\title{
Magnon dispersion in ferromagnetic semiconductors and metals under strong fields
}

\author{
L. C. M. Miranda* \\ Department of Physics, University of Arizona, Tucson, Arizona 85721 \\ and Departamento de Física, ${ }^{\dagger}$ Universidade de Brasilia, 70000 Brasília, D. F., Brazil
}

(Received 28 July 1975)

\begin{abstract}
The effect of strong magnetic fields on the magnon energy renormalization in an electron-magnon system is discussed. It is shown that under strong fields the magnon dispersion as well as the indirect exchange become anisotropic.
\end{abstract}

In a recent paper ${ }^{1}$ we reported on the effect of strong magnetic fields on the magnon damping in ferromagnetic semiconductors in connection with the problem of spin-wave amplification. As expected, external fields which change the spectrum and the occupation numbers of the electron states will influence the spectrum and damping of the spin waves. In fact, it was found in Ref. 1 that, at temperatures $k_{B} T \ll \epsilon_{F}, h \omega_{c}$ where $\epsilon_{F}$ and $\omega_{c}$ denote the Fermi energy and the electron cyclotron frequency, respectively, there exist bands of magnetic field in which the magnons are unstable in one band, stable in the next band, and so on. This result is completely opposed to that for weak applied fields ${ }^{2-5}$ in which case the amplification coefficient is found essentially independent of the external field. It is a consequence of the discreteness of the Landau levels of the electron states.

In the present paper we report on the effect of a strong magnetic field on the magnon dispersion relation, and consequently, on the indirect exchange, ${ }^{6-8}$ [Ruderman-Kittel-Kasuya-Yosida (RKKY) interaction] between the localized spins. Our model for a magnetic semiconductor and metal is that of an interacting conduction-electronlocalized-moment system. ${ }^{9-11}$ Within the framework of this simple model many properties of ferromagnetic semiconductors ${ }^{9,11}$ and metals ${ }^{10}$ have been at least qualitatively understood. In order that our predictions be valid the Landau levels must of course not be smeared out by temperature or collision broadening, $i_{\circ}$ e., the condition $\omega_{c} \tau>1$ must be satisfied, where $\tau$ is the electron scattering rate. In the case of ferro- magnetic semiconductors this condition entails restricting our choice to high-mobility materials. As discussed in Ref. 1, of the existing ferromagnetic semiconductors the best candidate for possible high-field studies is $\mathrm{CdCr}_{2} \mathrm{Se}_{4}$ doped with $\mathrm{Ag}$ for $\omega_{c} \tau>1$, for fields of about $90-100 \mathrm{kG}$. However, the results of the present paper are of little, if any, consequence for ferromagnetic semiconductors. The reason is that for these materials ${ }^{9}$ the contribution to the magnon dispersion relation due to the direct exchange between the localized spins is usually the dominant one. In contrast, for ferromagnetic metals ${ }^{10}$ the direct exchange plays no role. It then follows that our results are more appropriate to the case of magnetic metals or of magnetic atoms in a nonmagnetic metal host lattice.

The total Hamiltonian of the system comprises the conduction-electron part, the (direct) exchange-coupled-local-moment part, and the interaction term. We assume that the localized moments experience a ferromagnetic exchange interaction only with their nearest neighbors, and consider only the exchange part of the conduction-electron-local-moment interaction, which will be represented by a spin-dependent contact potential. Also, since we are interested in studying the system below the Curie temperature we shall introduce the magnon variables straightway. Finally, the effect of the external magnetic field on the carrier motion is taken into account by replacing the usual parabolic energy band by the Landau levels. Thus, in the second quantization formalism, the total Hamiltonian is given by ${ }^{1,9-11}$

$$
\begin{aligned}
H= & \sum_{\alpha \sigma} \epsilon_{\alpha \sigma} c_{\alpha \sigma}^{\dagger} c_{\alpha \sigma}+\sum_{\overrightarrow{\mathrm{k}}} \hbar \omega_{k} b_{k}^{\dagger} b_{k}-J_{s d}\left(\frac{S}{2 N}\right)^{1 / 2} \sum_{\alpha \beta \overrightarrow{\mathrm{k}}}\left(\left\langle\beta\left|e^{i \overrightarrow{\mathrm{k}} \cdot \overrightarrow{\mathrm{r}}}\right| \alpha\right\rangle c_{\beta_{t}}^{\dagger} c_{\alpha,} b_{k}\right. \\
& \left.+\left\langle\beta\left|e^{-i \overrightarrow{\mathrm{k}} \cdot \overrightarrow{\mathrm{r}}}\right| \alpha\right\rangle c_{\beta_{\mathrm{t}}}^{\dagger} c_{\alpha,} b_{k}^{\dagger}\right)+\frac{J_{S d}}{2 N} \sum_{\substack{\alpha \beta \\
\overrightarrow{\mathrm{k}} \overrightarrow{\mathrm{k}}^{\prime}}}\left\langle\beta\left|e^{i\left(\overrightarrow{\mathrm{k}}-\overrightarrow{\mathrm{k}}^{\prime}\right) \cdot \overrightarrow{\mathrm{r}}}\right| \alpha\right\rangle\left(c_{\beta_{\mathrm{t}}}^{\dagger} c_{\alpha,}-c_{\beta_{t}}^{\dagger}\right) b_{k^{\prime}}^{\dagger} b_{k} \cdot
\end{aligned}
$$

Here

$$
\epsilon_{\alpha \sigma}=\left(n+\frac{1}{2}\right) \hbar \omega_{c}+\hbar^{2} p_{z}^{2} / 2 m-\frac{1}{2}\left(\hbar \omega_{c}+J_{s d} s\right) \sigma
$$

and

$$
\hbar \omega_{k}=g \mu_{B} H+\hbar \omega_{k}^{(0)}=g \mu_{B} H+2 S I a^{2} k^{2}
$$


denote the electron and magnon energies, respectively, $\omega_{k}^{(0)}$ is the contribution from the direct exchange, and $\omega_{c}=e H / m c$. $J_{s d}$ is the exchange parameter between the localized spin and the conduction electron, $N$ is the number of magnetic atoms of ionic $g$-value $g, \mu_{B}$ is the Bohr magneton, $H$ is the external magnetic field assumed along the $z$ direction, $I$ is the (direct) exchange constant, and $a$ is the lattice spacing. $c_{\alpha \sigma}\left(c_{\alpha \sigma}^{\dagger}\right)$ is the usual annihilation (creation) operator for an electron in the state $\alpha\left(=n, p_{x}, p_{z}\right)$ with spin $\sigma$ in the Landau representation. ${ }^{12}$ Here $\sigma=+1$ for up conductionelectron moments, and $\sigma=-1$ for down moments. Finally, $b_{k}\left(b_{k}^{\dagger}\right)$ is the magnon annihilation (creation) operator.

Before continuing it should be noted that we are assuming that $J_{s d}$ is independent of the field. This is justifiable provided the $d$ orbitals are not explicitly dependent on the field and the $s$-electron wave function does not vary much over an ionic dimension (contact-potential approximation). The first point requires fields of the order of $10^{9} \mathrm{G}$ as can be seen from the theory of atoms in strong magnetic fields. ${ }^{13}$ The second condition requires $\left(\hbar / m \omega_{c}\right)^{1 / 2}$, the cyclotron radius, to be much larger than the lattice spacing $a$, i.e.,

$$
\left(\hbar / m \omega_{c}\right)^{1 / 2} \ll a \Rightarrow B \lesssim 10^{8} \mathrm{G} \text { 。 }
$$

Since the magnetic fields we have in mind are typically within the safe region of $90-100 \mathrm{kG}$, it is reasonable to neglect the field dependence as well as the more rigorous spatial dependence of the $s-d$ exchange integral.

The magnon self-energy, from which one obtains the magnon energy correction and lifetime, may be evaluated either by the thermodynamics Green's-function formalism ${ }^{11}$ or by the usual perturbation theory. ${ }^{14,15}$ We refer to Refs. 14 and 15 for a more detailed discussion. Up to second order in the coupling of the interaction, using Eqs. (1)-(3), the corresponding algebraic expression for the magnon energy correction is

$$
\begin{aligned}
\Sigma\left(\omega_{k}, \overrightarrow{\mathrm{k}}\right)= & \Sigma_{1}+\Sigma_{2}\left(\omega_{k}, \overrightarrow{\mathrm{k}}\right) \\
= & \frac{J_{s d}}{2 N} \sum_{\alpha}\left(f_{\alpha,}-f_{\alpha_{\iota}}\right)+\frac{J_{s d}^{2} S}{2 N} \\
& \times \sum_{\alpha, \beta}\left|\left\langle\alpha\left|e^{i \overrightarrow{\mathrm{k}} \cdot \overrightarrow{\mathrm{r}}}\right| \beta\right\rangle\right|^{2} \frac{f_{\alpha_{\iota}}-f_{\beta_{1}}}{\epsilon_{\alpha \downarrow}-\epsilon_{\beta_{\mathrm{t}}}-\hbar \omega_{k}},
\end{aligned}
$$

where $f_{\alpha \sigma}$ is the Fermi distribution function for an electron in the state $|\alpha, \sigma\rangle$. We shall use the step-function approximation for $f_{\alpha \sigma}$. The renormalized magnon energy $\hbar \tilde{\omega}_{k}$ is then determined by the solution of

$$
h \tilde{\omega}_{k}-g \mu_{B} H-h \omega_{k}^{(0)}-\Sigma\left(\tilde{\omega}_{k}, \vec{k}\right)=0 .
$$

In the following, the frequency dependence of the self-energy will be neglected. ${ }^{10}$ This is justified by the fact that the magnon effective mass $M=\hbar^{2} k^{2} / 2 \Sigma(0, \overrightarrow{\mathrm{k}})$ is much greater than the electron mass. Furthermore, we shall be mainly concerned with small values of $k$, and it can be shown that as $k \rightarrow 0$ the dynamic susceptibility tends to the static one.

Now, $\Sigma(0, \vec{k})$ may also be related to the RKKY indirect-exchange theory, ${ }^{16}$ modified by the presence of the strong field as follows. In secondorder perturbation theory in the $s-d$ coupling, following the steps of the RKKY theory, the effective exchange coupling between the localized spins $\overrightarrow{\mathrm{S}}_{i}$ and $\overrightarrow{\mathrm{S}}_{j}$ can be written as

$$
\begin{aligned}
J_{\text {eff }}(\overrightarrow{\mathrm{R}}= & \left.\overrightarrow{\mathrm{R}}_{i}-\overrightarrow{\mathrm{R}}_{j}\right) \\
= & -\left(\frac{V J_{s d}}{2 N}\right)^{2} \sum_{\alpha, \beta} \phi_{\beta}^{*}\left(\overrightarrow{\mathrm{R}}_{i}\right) \\
& \times \phi_{\alpha}\left(\overrightarrow{\mathrm{R}}_{i}\right) \phi_{\beta}\left(\overrightarrow{\mathrm{R}}_{j}\right) \phi_{\alpha}^{*}\left(\overrightarrow{\mathrm{R}}_{j}\right) \frac{f_{\alpha t}-f_{\beta t}}{\epsilon_{\alpha t}-\epsilon_{\beta t}},
\end{aligned}
$$

where $\phi_{\alpha}(\vec{r})$ is the Landau wave function. Taking the Fourier transform of $J_{\text {eff }}(\vec{R})$ we get

$$
\begin{aligned}
J_{\text {eff }}(k) & =\sum_{\overrightarrow{\mathrm{R}}} e^{-i \overrightarrow{\mathrm{k}} \cdot \overrightarrow{\mathrm{R}}} J_{\text {eff }}(\overrightarrow{\mathrm{R}}) \\
& =-\frac{J_{s d}^{2}}{4 N^{2}} \sum_{\alpha, \beta}\left|\left\langle\alpha\left|e^{i \overrightarrow{\mathrm{k}} \cdot \overrightarrow{\mathrm{r}}}\right| \beta\right\rangle\right|^{2} \frac{f_{\alpha \iota}-f_{\beta_{\mathrm{t}}}}{\epsilon_{\alpha \iota}-\epsilon_{\beta \mathrm{t}}} .
\end{aligned}
$$

On the other hand, $J_{\text {eff }}(k)$ leads to the following contribution to the magnon dispersion relation:

$$
2 S N\left[J_{\text {eff }}(0)-J_{\text {eff }}(k)\right]=\Sigma(0, k) .
$$

It then follows from Eqs. (6) and (7) that $J_{\text {eff }}(k)$ $=-\Sigma_{2}(0, k) / 2 S N$ and $\Sigma_{1}=-\Sigma_{2}(0, k=0)=2 S N J_{\text {eff }}(0)$ 。

The matrix element $\left\langle\alpha\left|e^{i \overrightarrow{\mathrm{k}} \cdot \overrightarrow{\mathrm{r}}}\right| \beta\right\rangle$ is calculated in Ref. 1 and we get

$$
\left|\left\langle\alpha\left|e^{i \overrightarrow{\mathrm{k}} \cdot \overrightarrow{\mathrm{r}}}\right| \beta\right\rangle\right|^{2}=\delta_{p_{x^{\prime}}, p_{x}+k_{x}} \delta_{p_{z}^{\prime}, p_{z^{\prime}}+k_{z}} \chi_{n^{\prime} n}(y),
$$

where $y=\frac{1}{2} k_{\perp}^{2} \sigma^{2}, \sigma=\left(\hbar / m \omega_{c}\right)^{1 / 2}$ is the cyclotron radius, and $k_{\perp}$ is the magnon wave vector perpendicular to the magnetic field. The function $\chi_{n^{\prime} n}(y)$ was defined in Ref. 1 . It is a sum of terms involving transitions between the Landau levels with $\Delta n \gtrless 0$. In the limit of small $k$ 's only the transitions with $\Delta n=0$ are important.

Substituting Eq. (8) into the expression for $\Sigma_{2}(0, k)$ obtained from Eq. (4), and performing the summations over $p_{x}$ and $p_{z}$, one obtains

$$
\Sigma_{2}(0, k)=\frac{S V k_{F} J_{s d}^{2}}{32 \pi^{2} \sigma^{2} N \epsilon_{F}} \sum_{n n^{\prime}} \chi_{n^{\prime} n}(y) F_{n^{\prime} n}\left(x_{11}\right),
$$

where

$$
x_{11}=k_{z} / 2 k_{F}
$$

and 


$$
F_{n^{\prime} n}\left(x_{\|}\right)=\frac{1}{x_{11}} \ln \frac{\left(1+\mu_{1}^{-}\right)\left(1-\mu_{2}^{-}\right)}{\left(1-\mu_{1}^{+}\right)\left(1+\mu_{2}^{+}\right)} .
$$

Here $k_{F}$ is the Fermi wave vector and

$$
\mu_{i}^{+}=\left(2 k_{i} k_{z} \pm k_{z}^{2}\right) / \bar{k}^{2} \text { with } i=1,2 ; \bar{k}^{2}=k_{2}^{2}-k_{1}^{2},
$$

$k_{1}^{2}=k_{F}^{2}-\left(n^{\prime}+\frac{1}{2}\right) \frac{2}{\sigma^{2}}-\frac{m}{\hbar^{2}}\left(\hbar \omega_{c}+J_{s d} S\right)$,

and

$$
k_{2}^{2}=k_{F}^{2}-\left(n+\frac{1}{2}\right) \frac{2}{\sigma^{2}}+\frac{m}{\hbar^{2}}\left(\hbar \omega_{c}+J_{s d} S\right) .
$$

In the limit of long wavelengths $\left(k \ll k_{F}, \sigma^{-1}\right)$, expanding $F_{n^{\prime} n}\left(x_{\| 1}\right)$ and $\chi_{n^{\prime} n}(y)$ in powers of $x_{11}$ and $y$, respectively, Eq. (9) leads to a quadratic magnon dispersion of the form

$$
\Sigma(0, k)=\frac{3 S}{4} \frac{N_{e}}{N} \frac{J_{s d}^{2}}{\epsilon_{F}}\left(x_{\perp}^{2}+\gamma^{2} x_{\|}^{2}\right),
$$

where

$$
x_{\perp}=k_{\perp} / 2 k_{F}, \quad \gamma=2\left(\hbar \omega_{c} \epsilon_{F}\right)^{1 / 2} / J_{s d} S
$$

and $N_{e}$ is the number of conduction electrons. In arriving at Eq. (13) we have approximated $\chi_{n^{\prime} n}(y)$ by the term involving only $n^{\prime}=n$, which is valid as long as $k_{\perp} v_{F} \ll \omega_{c}$, where $v_{F}$ is the Fermi velocity. It was also assumed that $J_{s d} S$ is greater than $\hbar \omega_{c}$ and that only the lowest Landau levels are important.

Equation (13) indicates that in the presence of strong fields the magnon dispersion becomes anisotropic. This is of course a consequence of the Landau level structure. In particular, the magnon dispersion along the magnetic field axis is enhanced with respect to the dispersion in the perpendicular direction. This result could have been anticipated since under strong fields the electron motion perpendicular to the field becomes localized, which makes it more difficult to create a spin deviation which propagates in that direction. In terms of the indirect-exchange theory this could alternatively be seen as due to the localization of the indirect-exchange integral in the perpendicular direction, as we shall see below. Finally, in the limit of weak or zero magnetic field, Eq. (13) reduces to the well-known result of the RKKY theory, namely, ${ }^{10,16}$

$$
\Sigma^{(H=0)}(0, k)=\frac{S}{2} \frac{N_{e}}{N} \frac{J_{s d}^{2}}{\epsilon_{F}} x^{2}
$$

To see this we note that as $H \rightarrow 0$ the term in $x_{11}$ vanishes at the same time that $x_{1}^{2} \rightarrow \frac{2}{3} x^{2}\left(x^{2}=x_{1}^{2}\right.$ $\left.+x_{11}^{2}\right)$, since the system now becomes isotropic. To conclude, let us now analyze these results in terms of the indirect exchange. As mentioned before, $J_{\text {eff }}(\vec{R})$ may be obtained directly from $E q$. (9) as

$$
J_{\text {eff }}(\overrightarrow{\mathrm{R}})=-\frac{v}{2 S N(2 \pi)^{3}} \int d^{3} k e^{i \overrightarrow{\mathrm{k}} \cdot \overrightarrow{\mathrm{R}}} \Sigma_{2}(0, \overrightarrow{\mathrm{k}})
$$

Substituting $e^{i \overrightarrow{\mathrm{k}} \cdot \overrightarrow{\mathrm{R}}}$ by

$$
e^{i k_{\|} R_{\|}}+\sum_{\nu=-\infty}^{\infty} i^{\nu} J_{\nu}\left(k_{\perp} R_{\perp}\right) e^{i \nu\left(\phi_{k}-\phi\right)}
$$

and assuming the same approximations as those involved in arriving at Eq. (13), one finally gets

$$
J_{\text {eff }}(R)=\frac{m V^{2} J_{s d}^{2}}{(2 \pi)^{3} 2 \hbar^{2} \sigma^{4} N^{2}} e^{-R_{\perp}^{2} / 2 \sigma^{2}} \operatorname{si}\left(2 k_{F}\left|R_{\|}\right|\right),
$$

where $\operatorname{si}(x)$ is the sine-integral function. ${ }^{17}$ Once again we see that, contrary to the zero-field case, the indirect exchange in strong fields is not isotropic, being oscillatory in the direction parallel to the field and relatively localized in the perpendicular direction. Furthermore, Eq. (16) leads to the same type of oscillatory magnetic ordering as in the case of the usual RKKY theory, i。e., $J_{\text {eff }}(\vec{R})$ is ferromagnetic for $2 k_{F}\left|R_{\|}\right|$less than the first zero of $\operatorname{si}(x)$, which occurs at $x=1.9$. Comparing this value of the first zero of $J_{\text {eff }}(\vec{R})$ with that of the RKKY theory, namely $=4.5$, one concludes that the effect of the strong field is not only that of localizing the indirect exchange in the perpendicular direction but also of confining the oscillatory part of the interaction. In fact using the properties of $\operatorname{si}(x),{ }^{17}$ it is easy to see that $J_{\text {eff }}(\vec{R})$ as given by $\mathrm{Eq}$. (16) vanishes more rapidly than its counterpart in the zero-field case.

Finally, we note the following in regard to the size of the effects reported in this note. As mentioned before, the anisotropy induced by the Landau levels will hardly be seen in ferromagnetic semiconductors, the reason being that in these materials the direct exchange is usually far greater than the indirect exchange as given by the RKKY theory. On the other hand, from Eqs. (13) and (15) we see that the magnon energy correction is just about the same as that of RKKY theory. Hence, possible experimental observations are therefore restricted to the cases of ferromagnetic metals (rare-earth metals) and of magnetic impurities in a nonmagnetic metal host.

The author is very grateful to Professor W. E. Lamb, Jr. for his hospitality and for making possible the author's visit to the University of Arizona. 
*John Simon Guggenheim Fellowship.

${ }^{\dagger}$ Permanent address.

${ }^{1}$ M. A. F. Gomes and L. C. M. Miranda, Phys. Rev. B 12,3788 (1975).

${ }^{2}$ M. D. Coutinho, Jr., L. C. M. Mirando, and S. M. Rezende, Phys. Status Solidi 57, 85 (1973).

${ }^{3}$ M. D. Coutinho, Jr., L. C. M. Miranda, and S. M. Rezende, Phys. Status Solidi 65,689 (1974).

${ }^{4}$ M. D. Coutinho, Jr., L. C. M. Miranda, and S. M. Rezende, Phys. Status Solidi 66, 395 (1974).

${ }^{5}$ L. C. M. Miranda, Phys. Status Solidi 60,617 (1973).

${ }^{6}$ M. A. Ruderman and C. Kittel, Phys。 Rev。 96,99 (1954).

${ }^{7} \mathrm{~T}$. Kasuya, Prog. Theor. Phys. 16, 45 (1956).

${ }^{8} \mathrm{~K}$. Yosida, Phys. Rev. 106, 893 (1957).

${ }^{9} \mathrm{~S}$. Methfessel and D. C. Mattis, in Handbuch der Phys-

$i k$, edited by S. Flügge (Springer-Verlag, Berlin,

1968), Vol. XVIII/1.

${ }^{10} \mathrm{~S}$. V. Vonsovski and Yu. A. Yzyumov, Usp. Fiz. Nauk 78, 3 (1963) [Sov. Phys. -Usp. 5, 723 (1963)].

${ }^{11}$ R. B. Woolsey and R. M. White, Phys. Rev. B 1 , 4474 (1970).

${ }^{12}$ See, e.g. , L. D. Landau and E. M. Lifshitz, Quantum Mechanics (Pergamon, Oxford, 1958), p. 424.

${ }^{13}$ H. S. Brandi, Phys. Rev. A 11, 1835 (1975).

${ }^{14}$ L. C. Davis and S. H. Liu, Phys. Rev. 163,503 (1967)。

${ }^{15}$ H. S. D. Cole and R. E. Turner, Phys. Rev. Lett. 19, 501 (1967).

${ }^{16}$ S. H. Liu, Phys. Rev. 123, 470 (1961).

${ }^{17}$ See, e.g. , Handbook of Mathematical Functions, edited by M. Abramowitz and I. A. Stegun (U. S. GPO, Washington, D. C., 1964); p. 231. 